\title{
Not a Routine Case of Recurrent Cellulitis: Well's Syndrome
}

\author{
Jeffrey J. Wargo
}

\begin{abstract}
When a 28-year-old man with suspected recurrent right forearm cellulitis did not respond to anti-microbial therapy, a dermatological consultation with cutaneous biopsy was obtained. Histopathology along with correlation of his clinical course confirmed a diagnosis of Well's syndrome (eosinophilic cellulitis) and he was successfully treated with oral anti-histamines and topical corticosteroids. This case emphasizes the importance of consideration for non-infectious etiologies of recurrent cellulitic lesions that do not respond to typical anti-microbial therapy.
\end{abstract}

Keywords: Well's syndrome; Eosinophilic cellulitis; Recurrent cellulitis

\section{Introduction}

Eosinophilic cellulitis, also known as Wells' syndrome, is a recurrent granulomatous dermatitis associated with peripheral eosinophila [1]. This rare cutaneous condition appears cellulitic, lacks systemic involvement, does not respond to antibiotics, requires a high degree of clinical suspicion to diagnose, and is managed most effectively with oral corticosteroids. With the increasing threat of antibiotic resistance and importance of antimicrobial stewardship, it is imperative to correctly differentiate cellulitis from non-infectious cutaneous conditions, employ a comprehensive differential diagnosis for erythematous plaques, and understand when to perform a skin biopsy to aid with diagnosis. We present a case of Well's syndrome in a patient admitted to our observation unit for "recurrent cellulitis".

\section{Case Report}

\section{Presentation}

A 28-year-old man presented with suspected recurrent right forearm cellulitis. The patient had had four episodes of pru-

Manuscript accepted for publication October 26, 2016

Department of Internal Medicine, University of Michigan Medical School, 1500 East Medical Center Drive, Ann Arbor, MI 48109, USA.

Email: jwarg75@gmail.com

doi: http://dx.doi.org/10.14740/jmc2680w ritic, edematous, erythematous plaques on the arms and neck over the past 3 months. Each episode lasted for 7 - 10 days and was in a different location. He was treated successfully with antibiotics, but this episode developed while on clindamycin. He had a history of HIV managed with daily emtricitabine, tenofovir, and rilpivirine. His recent CD4 count was 1,204 cells $/ \mathrm{mm}^{3}$ with an undetectable viral load. He was started on IV cefazolin in the emergency department and admitted to the observation unit.

\section{Assessment and diagnosis}

On admission, he had a temperature of $36.8^{\circ} \mathrm{C}$, blood pressure of 134/77 mm Hg, heart rate of 96 beats per minute, respiratory rate of 15 breaths per minute, and an oxygen saturation of $96 \%$ on room air. On his right forearm and inner upper arm, there were several warm erythematous slightly edematous plaques (Fig. 1a). Laboratory analysis was significant for a white blood cell count of $15,200 / \mathrm{uL}$ with $80.4 \%$ neutrophils and $5 \%$ eosinophils. As the patient's rash did not improve on antibiotics, dermatology was consulted and a biopsy was performed. Histopathology revealed perivascular and interstitial infiltrate with numerous eosinophils and neutrophils, and prominent superficial papillary dermal edema suggesting a diagnosis of Well's syndrome (eosinophilic cellulitis) (Fig. 1b). The patient was successfully treated with anti-histamines and topical corticosteroids.

\section{Discussion}

When a physician is challenged with a case of cellulitis that does not respond to conventional antibiotic therapy, non-infectious etiologies must be considered. After a thorough history, physical, and diagnostic workup has been completed, skin biopsy with histopathology, culture, and direct immunofluorescence may be the only diagnostic option left to secure a diagnosis. In our case, we considered arthropod assault, acute febrile neutrophilic dermatosis, contact dermatitis, Wells' syndrome, dermal hypersensitivity reaction, urticaria, erythema migrans, toxin-mediated erythema, cellulitis, and drug reactions. Thrombophlebitis, gouty arthritis, familial Mediterranean fever, panniculitis, toxic erythema of chemotherapy, angioedema, and foreign-body reactions represent diseases that commonly masquerade as infectious cellulitis as well [2].

Eosinophilic cellulitis, also known as Wells' syndrome, was first described in 1971 by Dr. Wells as a recurrent granu- 


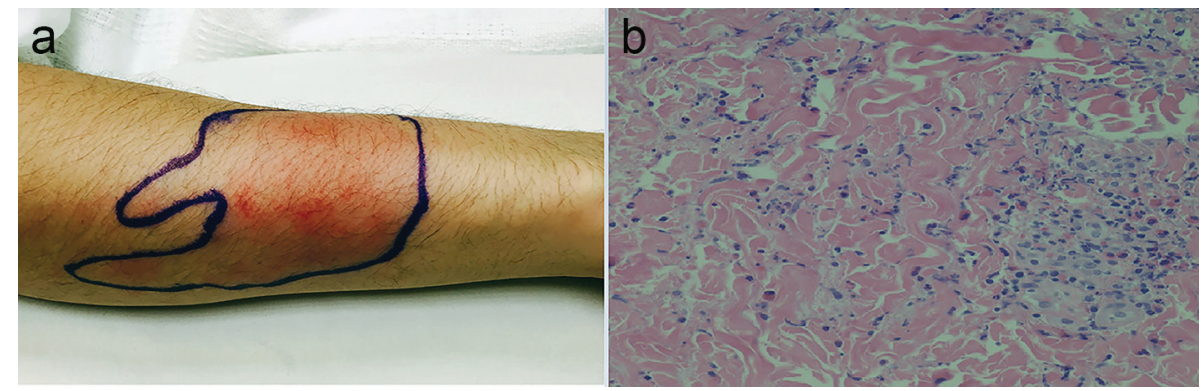

Figure 1. (a) A warm erythematous slightly edematous plaque on the right forearm. (b) Perivascular and interstitial infiltrate with numerous eosinophils, neutrophils, and prominent superficial papillary dermal edema.

lomatous dermatitis with eosinophilia [1]. This rare cutaneous condition lacks systemic involvement, does not respond to antibiotics, requires a high degree of clinical suspicion to diagnose, and presents with peripheral eosinophilia in only about $50 \%$ of cases [3]. Cutaneous findings appear cellulitic and most typically present with erythematous plaques preceded by pruritis, but blisters, bullae, or nodules may also be seen. Resolution of the lesions occurs in 2 - 8 weeks and may result in hyperpigmentation and skin atrophy resembling morphea [4]. Acute histology reveals tissue edema and a dermal eosinophilic infiltration in a perivascular pattern, while subacute to chronic histology reveals the classic "flame figures" composed of bundles of collagen coated with eosinophils [5]. As flame figures have been associated with other conditions such as Churg-Strauss, spider bites, parasitic infections, follicular mucinosis, and herpes gestationis, a clinical-pathologic approach is required to confirm the diagnosis. Well's syndrome may be associated with infectious diseases, arthropod assault, malignancy, immunologic disorders, thimerosal-containing vaccines, or medications, but a specific etiology and pathogenesis has not been determined. A literature review of 32 cases of idiopathic eosinophilic cellulitis in 2012 concluded that oral steroids achieved the highest rate of resolution and were $92 \%$ effective. In these patients, oral prednisone was typically given $2 \mathrm{mg} / \mathrm{kg}$ for 1 week and then tapered over 2 - 3 weeks. Topical steroids and oral anti-histamines were less effective. As recurrence occurs in up to half of cases, treatment of the underlying associated condition (if determined) is imperative, and alternate day low dose prednisone may be indicated.

\section{Conflicts of Interest}

There are no conflicts of interest to report.

\section{Funding}

None.

\section{References}

1. Wells GC. Recurrent granulomatous dermatitis with eosinophilia. Trans St Johns Hosp Dermatol Soc. 1971;57(1):46-56.

2. Falagas ME, Vergidis PI. Narrative review: diseases that masquerade as infectious cellulitis. Ann Intern Med. 2005;142(1):47-55.

3. Caputo R, Marzano AV, Vezzoli P, Lunardon L. Wells syndrome in adults and children: a report of 19 cases. Arch Dermatol. 2006;142(9):1157-1161.

4. Sinno H, Lacroix JP, Lee J, Izadpanah A, Borsuk R, Watters K, Gilardino M. Diagnosis and management of eosinophilic cellulitis (Wells' syndrome): A case series and literature review. Can J Plast Surg. 2012;20(2):91-97.

5. Beer TW, Langtry JA, Phillips WG, Wojnarowska F. Flame figures in bullous pemphigoid. Dermatology. 1994;188(4):310-312. 